\title{
Jacobi forms over imaginary quadratic number fields
}

\author{
by \\ Howard Skogman (Los Angeles, CA)
}

1. Introduction. The functions considered in this paper are generalizations of the classical Jacobi forms. Classical Jacobi forms are functions satisfying two transformation properties

$$
\phi\left(\frac{a \tau+b}{c \tau+d}, \frac{z}{c \tau+d}\right)=(c \tau+d)^{k} e^{2 \pi i m \frac{c z^{2}}{c \tau+d}} \phi(\tau, z)
$$

and

$$
\phi(\tau, z+\lambda \tau+\mu)=e^{-2 \pi i m\left(\lambda^{2} \tau+2 \lambda z\right)} \phi(\tau, z)
$$

for all matrices $\left(\begin{array}{ll}a & b \\ c & d\end{array}\right)$ in $\mathrm{Sl}_{2}(\mathbb{Z})$, and vectors $[\lambda, \mu]$ in $\mathbb{Z}^{2}$. The original examples of such forms were created by Jacobi in [4]. These examples were types of theta functions of quadratic forms. For example, given a positive definite $l \times l$ matrix $Q$ with rational integer entries and even diagonal entries, and given a fixed vector $\vec{b}$ in $\mathbb{Z}^{l}$, define the function

$$
\theta_{Q, b}(\tau, z)=\sum_{\vec{r} \in \mathbb{Z}^{l}} e^{\pi i^{t} \vec{r} Q \vec{r} \tau+2^{t} \vec{r} Q \vec{b} z}
$$

These theta functions have the special transformation properties described above; however, there was no investigation into general functions satisfying the transformation properties presented above until [1].

The functions studied in this paper are generalizations of these classical Jacobi forms where the ring of rational integers $\mathbb{Z}$ is replaced by the ring of integers in an imaginary quadratic field. There have been a few investigations into this area. Gritsenko in [2] studied functions which satisfied generalizations of the transformation formulas where the invariance properties were with respect to all $\left(\begin{array}{ll}a & b \\ c & d\end{array}\right)$ in $\operatorname{Sl}_{2}(\mathbb{Z}[\sqrt{-1}])$ and $[\lambda, \mu]$ in $\mathbb{Z}[\sqrt{-1}]^{2}$. Krieg in [5] studied similar forms with the ring $\mathbb{Z}[\sqrt{-1}]$ replaced by the ring of integers in any imaginary quadratic field, and studied their connection to symplectic modular forms. Haverkamp in [3] studied forms where the first transformation properties are with respect to $\mathrm{Sl}_{2}(\mathbb{Z})$ but the second is with respect to vectors in the ring of integers in an imaginary quadratic field.

2000 Mathematics Subject Classification: Primary 11F55; Secondary 11F41, 11F27. 
One difference between the cited papers and this paper is the use of quaternionic variables. This causes some problems because the quaternions are non-commutative. This non-commutativity also creates the need to introduce an index pair or an index vector. The $m$ which appears in the classical transformation formulas is replaced by the inner product of a vector with itself which arises from the quadratic forms used in the construction. The difference is that instead of all of the factors having the inner product of the vector with itself, some appear with the inner product of the vector and its complex conjugate. This index vector does not appear in Jacobi forms of totally real fields (see [9]). The results in [9] along with those in this paper give a construction of Jacobi forms for any algebraic number field which is presented in [8].

The construction relies on extending the connection between Jacobi forms and symplectic modular forms to algebraic number fields. The actual construction produces the "natural" generalization of Jacobi's initial examples. Another interesting difference between this work and [2], [5] is the use of a different symplectic group. The cited works all used the Hermitian symplectic group which is all matrices $M$ with entries in the ring of integers in the field such that ${ }^{t} \bar{M} J M=J=\left(\begin{array}{cc}0 & -I_{n} \\ I_{n} & 0\end{array}\right)$ where the bar denotes complex conjugation. The symplectic group employed here has a similar requirement without the bar on the first matrix.

1.1. Some notation. Throughout the paper some standard notation will be used which is listed here. $\mathbb{Q}, \mathbb{Z}, \mathbb{R}, \mathbb{C}$ will be used to denote the rational numbers, the rational integers, the real numbers, and the complex numbers respectively. We will use $K$ to denote an imaginary quadratic number field and $\mathcal{O}_{K}$ will be the ring of algebraic integers in this number field. We will denote by $\delta_{K}, \delta_{K}^{-1}$ the different and the inverse different of the field $K$. The $n \times n$ matrices with entries in $\mathbb{F}$ is denoted $M_{n}(\mathbb{F})$. Given a matrix $M \in M_{n}(\mathbb{F})$ the transpose of $M$ will be denoted ${ }^{t} M$. The $l \times l$ identity matrix will be $I_{l}$. The imaginary part of $\alpha$ is denoted $\operatorname{Im} \alpha$. The $n$-dimensional $\mathbb{Z}$-module spanned by the $\left\{a_{i}\right\}_{1 \leq i \leq n}$ is written $\left[a_{1}, \ldots, a_{n}\right]_{\mathbb{Z}}$. For $a \in \mathbb{C}, \bar{a}$ is used to mean the usual complex conjugate. We also use $e[\ldots]$ to mean $e^{2 \pi i[\ldots]}$. The symplectic upper half space of genus $n$ will be written $\mathfrak{h}^{(n)}=$ $\left\{Z=X+i Y \in M_{n}(\mathbb{C}) \mid{ }^{t} Z=Z, Y>0\right\}$ which is all symmetric complex matrices with positive definite imaginary part.

2. Jacobi forms over $\mathbb{Q}$. All of the results presented in this section are presented in [1].

The Jacobi group is $\Gamma^{\mathrm{J}}(\mathbb{Z})=\mathrm{Sl}_{2}(\mathbb{Z}) \ltimes \mathbb{Z}^{2}$ and the space on which this group acts is $\mathfrak{h} \times \mathbb{C}$ where $\mathfrak{h}:=\{z \in \mathbb{C}, \operatorname{Im} z>0\}$ and $\mathbb{C}$ is the field of complex numbers. The actions of $\Gamma^{\mathrm{J}}(\mathbb{Z})$ on $\mathfrak{h} \times \mathbb{C}$ are for $M$ in $\mathrm{Sl}_{2}(\mathbb{Z})$, 


$$
M \circ(\tau, z)=\left(\begin{array}{ll}
a & b \\
c & d
\end{array}\right) \circ(\tau, z)=\left(\frac{a \tau+b}{c \tau+d}, \frac{z}{c \tau+d}\right)
$$

and for $[\lambda, \mu]$ in $\mathbb{Z}^{2}$,

$$
[\lambda, \mu] \circ(\tau, z)=(\tau, z+\lambda \tau+\mu)
$$

The general element of this Jacobi group acts first by the matrix and then by the vector which insures there is a group action on this space.

Definition. A function $\phi(\tau, z): \mathfrak{h} \times \mathbb{C} \rightarrow \mathbb{C}$ that satisfies

$$
\begin{aligned}
\phi\left(\frac{a \tau+b}{c \tau+d}, \frac{z}{c \tau+d}\right) & =(c \tau+d)^{k} e^{2 \pi i m \frac{c z^{2}}{c \tau+d}} \phi(\tau, z), \\
\phi(\tau, z+\lambda \tau+\mu) & =e^{-2 \pi i m\left(\lambda^{2} \tau+2 \lambda z\right)} \phi(\tau, z)
\end{aligned}
$$

for all $\left(\begin{array}{ll}a & b \\ c & d\end{array}\right) \in \mathrm{Sl}_{2}(\mathbb{Z}),[\lambda, \mu] \in \mathbb{Z}^{2}$, and is analytic in both variables is called a Jacobi form of weight $k$ and index $m$.

Because of these formulas, a Jacobi form satisfies the equalities $\phi(\tau+1, z)$ $=\phi(\tau, z+1)=\phi(\tau, z)$ and therefore possesses a Fourier expansion with respect to both $\tau$ and $z$, i.e.

$$
\phi(\tau, z)=\sum_{n=0}^{\infty} \sum_{r \in \mathbb{Z}, r^{2} \leq 4 n m} c(n, r) q^{n} \xi^{r}
$$

where $q=e^{2 \pi i \tau}, \xi=e^{2 \pi i z}$. Note that the $r^{2} \leq 4 n m$ is a condition to make the function analytic at infinity. In the definition of Jacobi forms one may also restrict to a subgroup of the Jacobi group, and one may allow a multiplier system in the first transformation formula. Specifically we may replace (5) by

$$
\phi\left(\frac{a \tau+b}{c \tau+d}, \frac{z}{c \tau+d}\right)=\chi(M)(c \tau+d)^{k} e^{2 \pi i m \frac{c z^{2}}{c \tau+d}} \phi(\tau, z)
$$

where $\chi(M)$ is a root of unity depending only on the matrix $M$.

2.1. The Jacobi group for imaginary quadratic number fields. The Jacobi group of the imaginary quadratic number field $K$ will be denoted $\Gamma^{\mathrm{J}}(K)$ where

$$
\Gamma^{\mathrm{J}}(K)=\mathrm{Sl}_{2}\left(\mathcal{O}_{K}\right) \ltimes \mathcal{O}_{K}^{2}
$$

This group acts on

$$
\mathcal{H}=\mathfrak{h}^{(\mathcal{Q})} \times \mathcal{Q}
$$

where $Q$ is used to denote

$$
\mathcal{Q}=\left\{x+y \kappa \mid x, y \in \mathbb{C}, \kappa^{2}=-1, a \kappa=\kappa \bar{a}, \forall a \in \mathbb{C}\right\}
$$

which is known as the full ring of quaternions. The upper half plane of quaternions is denoted $\mathfrak{h}^{(\mathcal{Q})}=\left\{x+y \kappa \in \mathcal{Q} \mid y \in \mathbb{R}^{+}\right\}$which may also be written as all quaternions that have no $j$ component and positive $\kappa$ 
component. It will be crucial in all of the following calculations to be careful about the ordering of elements of the quaternions since elements of this ring do not commute. Variables of this space will be listed as $(\tau, z) \in \mathfrak{h}^{(\mathcal{Q})} \times \mathcal{Q}$. These variables will be represented as $\tau=x+y \kappa \in \mathfrak{h}^{(\mathcal{Q})}$, and $z=u+v \kappa \in \mathcal{Q}$. The actions of $\Gamma^{\mathrm{J}}\left(\mathcal{O}_{K}\right)$ on the space $\mathcal{H}$ are given by, for $\left(\begin{array}{cc}\alpha & \beta \\ \gamma & \delta\end{array}\right) \in \operatorname{Sl}_{2}\left(\mathcal{O}_{K}\right)$,

$$
\begin{gathered}
\left(\begin{array}{cc}
\alpha & \beta \\
\gamma & \delta
\end{array}\right) \circ(\tau, z)=\left((\alpha \tau+\beta)(\gamma \tau+\delta)^{-1},(\tau \gamma+\delta)^{-1} z\right), \\
\forall[\lambda, \mu] \in \mathcal{O}_{K}^{2}, \quad[\lambda, \mu] \circ(\tau, z)=(\tau, z+\tau \lambda+\mu) .
\end{gathered}
$$

As before, a general element of the Jacobi group acts first by the matrix and then by the vector. These actions are natural generalizations of the classical actions up to ordering of elements.

The definition of congruence subgroups as above may be extended to $K$ and thereby define subgroups of the Jacobi group using subgroups of $\mathrm{Sl}_{2}\left(\mathcal{O}_{K}\right)$ and sublattices in the ring of integers. For example, extending the earlier notation, given an ideal $\mathfrak{N} \subseteq \mathcal{O}_{K}$ define

$$
\Gamma_{0}(\mathfrak{N})=\left\{M=\left(\begin{array}{ll}
a & b \\
c & d
\end{array}\right), M \in \mathrm{Sl}_{2}\left(\mathcal{O}_{K}\right) \text { and } c \in \mathfrak{N}\right\},
$$

and so a subgroup of $\Gamma^{\mathrm{J}}\left(\mathcal{O}_{K}\right)$ of the form $\Gamma_{0}(\mathfrak{N}) \ltimes \mathfrak{a}^{2}$ for ideals $\mathfrak{a}, \mathfrak{N} \subseteq \mathcal{O}_{K}$ may be used in place of $\Gamma^{\mathrm{J}}\left(\mathcal{O}_{K}\right)$.

2.2. Jacobi forms over imaginary quadratic number fields. In order to define Jacobi forms over $K$ it is necessary to define the transformation formulas and transformation factors for the Jacobi group. It will be useful to define an exponential of a quaternion as

$$
e[a+b \kappa]=e[a+\bar{a}+i(b+\bar{b})]=e^{2 \pi i(a+\bar{a}+i[b+\bar{b}])}, \quad \forall a+b \kappa \in \mathcal{Q} .
$$

To reduce some notation in the formulas the factor $\mathcal{N}(\gamma \tau+\delta)$ is used to denote the factor that replaces the $(c \tau+d)$ in the classical transformation formula (5), so denote for $\tau$ in $\mathfrak{h}^{(\mathcal{Q})}, \gamma, \delta$ in $\mathcal{O}_{K}$

$$
\mathcal{N}(\gamma \tau+\delta)=\left(\left|\gamma x_{j}+\delta\right|^{2}+y_{j}^{2}|\gamma|^{2}\right) .
$$

This is simply the quaternionic norm where the usual complex norm is denoted by $|\ldots|$, i.e. $|a+b i|^{2}=a^{2}+b^{2}$.

In the case of number fields, especially fields with complex conjugates, it is necessary to define the index vector associated with the index $\mathbf{m}$. The index vector will be a certain complex vector, denoted $\vec{m}$, of length equal to twice the weight $k$ of the form, such that ${ }^{t} \vec{m} \vec{m}=\mathbf{m}$. The explicit nature and the appearance of the index vector will be made clear in the construction. 
Definition. A Jacobi form of weight $k$ and index $\mathbf{m}$ and index vector $\vec{m}$ for the imaginary quadratic number field $K$ is a function

$$
\Phi(\tau, z): \mathfrak{h}^{(\mathcal{Q})} \times \mathcal{Q} \rightarrow \mathbb{C}
$$

satisfying

$$
\begin{aligned}
& \Phi\left(\left(\begin{array}{ll}
\alpha & \beta \\
\gamma & \delta
\end{array}\right) \circ(\tau, z)\right) \\
& =\chi\left(\begin{array}{ll}
\alpha & \beta \\
\gamma & \delta
\end{array}\right) \mathcal{N}(\gamma \tau+\delta)^{k} e\left[{ }^{t} \vec{m}(u+\bar{v} \kappa)(\gamma \tau+\delta)^{-1} \gamma(u+v \kappa) \vec{m}\right] \Phi(\tau, z)
\end{aligned}
$$

and

$$
\Phi([\lambda, \mu] \circ(\tau, z))=e\left[-{ }^{t} \vec{m}(\lambda \tau \lambda+2 \lambda z) \vec{m}\right] \Phi(\tau, z)
$$

for all $\left(\begin{array}{cc}\alpha & \beta \\ \gamma & \delta\end{array}\right) \in \operatorname{Sl}_{2}\left(\mathcal{O}_{K}\right),[\lambda, \mu] \in \mathcal{O}_{K}^{2}$, where $\chi$ is a root of unity depending only on the matrix.

It is important to note that the forms which will be created have weight $k$ in $\mathbb{Z}$ or in $\mathbb{Z}+1 / 2$, however the index $\mathbf{m}$ is allowed to be any integer in the field. These transformation formulas are easily seen to be generalizations of the classical case. This definition may be restricted to subgroups of the Jacobi group. For the forms constructed here, the root of unity $\chi$ depending on the matrix will be an eighth root of unity, i.e. $\chi(M)^{8}=1$ for all $M$ in $\mathrm{Sl}_{2}\left(\mathcal{O}_{K}\right)$ which may be determined using work of Stark in [10]. Note there is no multiplier system in the second transformation formula (11). The group action by elements of the form $[\lambda, \mu]$ along with the fact that theta functions will be used in the construction, force the multiplier system to be trivial on these Jacobi group elements. The only differences from the exact form of the classical formulas and factors results from the non-commutative nature of the quaternionic variables. It is also useful to note that the index vector is only necessary for fields with complex conjugates. So for totally real fields it is only required to specify the index and not the index vector. Also for totally real fields, it is possible to require that the Jacobi forms be analytic and in fact this will be necessary in order to prove the connection to vector valued modular forms again (see [9]). However, for the general algebraic number field there is no notion of an analytic function so it is left out of the definition.

3. Construction. In this section a construction will be outlined that produces Jacobi forms of weight $1 / 2$, indexes of the form $\lambda^{2}$, and index vectors of the form $(\lambda)$, for $\lambda$ in $\mathcal{O}_{K}$. There is a method of producing arbitrary half-integral weights using quadratic forms which we will delay until later and suggest [7] for more information. The idea of the construction is to use the relationship between Jacobi forms and symplectic modular forms. 
Specifically, first create a symplectic modular form which transforms over a number field, then take a Fourier expansion with respect to the appropriate variables and the Fourier coefficients will be Jacobi forms. The specific function we will create as a first example is a generalization of the classical Jacobi theta function

$$
\theta_{1}(\tau, z)=\sum_{n \in \mathbb{Z}} e^{\pi i\left(n^{2} \tau+2 n z\right)},
$$

which is a Jacobi form of weight $1 / 2$ and index 1 on a subgroup of the Jacobi group $\Gamma^{\mathrm{J}}(\mathbb{Z})$.

3.1. The index vector. To introduce the index vector, it is easiest to describe how it appears. First, one creates a more general type of Jacobi theta function for any symmetric, positive definite quadratic form $Q$. That is, if $Q$ is an $l$-dimensional symmetric quadratic form over the rational integers with even diagonal entries, i.e. for $a$ in $\mathbb{Z}^{l}, Q(a)={ }^{t} a Q a$ and $Q(a)>0$, for all $a$ in $\mathbb{Z}^{l}, a \neq 0$, then fix a vector $b$ in $\mathbb{Z}^{l}$ and define

$$
\theta_{Q}(\tau, z)=\sum_{a \in \mathbb{Z}^{l}} e^{\pi i\left({ }^{t} a Q a \tau+2^{t} a Q b z\right)}
$$

which is a Jacobi form of weight $l / 2$ and index $Q(b)$ possibly over a subgroup of $\Gamma^{\mathrm{J}}(\mathbb{Z})$. The natural generalization of this form may be given for an $l$ dimensional symmetric quadratic form $Q_{K}$ whose entries are in $\mathcal{O}_{K}$. It is known that such a form decomposes as $Q_{K}={ }^{t} L L$, and for a fixed $b$ in $\mathcal{O}_{K}^{l}$ the general Jacobi theta function of a quadratic form may be written as

$$
\theta_{Q_{K}, b}(\tau, z)=\sum_{a \in \mathcal{O}_{K}^{l}} \exp \left(\pi i\left({ }^{t} a^{t} L \tau L a+2{ }^{t} a{ }^{t} L z L b\right)\right),
$$

where we are using the previously defined exponential for quaternions. Such a form will be constructed and shown to be a Jacobi form over $K$ of weight $l / 2$ and index ${ }^{t} b Q_{K} b={ }^{t} b^{t} L L b$, except that the non-complex parts of the quaternionic transformation factors will have an index of ${ }^{t} b^{t} L \overline{L b}$. So if the in$\operatorname{dex} \mathbf{m}=\sum_{i=1}^{l} c_{i}^{2}$ the non-complex parts of the quaternionic transformation factors have an index of $\sum_{i=1}^{l} c_{i} \bar{c}_{i}$. This creates the need to specify the index vector which is $\vec{m}=L b$ in order to completely specify the transformation factors. Actually knowing the index vector is overkill. The transformation formulas are completely determined by the index pair $Q(b)$ and the index for the non-complex part of the quaternionic factors ${ }^{t} b^{t} L \overline{L b}$. So if another index vector has the same index pair as $L b$, the Jacobi form with this index vector will transform identically to the Jacobi form with the index vector $L b$.

This means that instead of requiring that forms have the same index vector, the definition of Jacobi forms over $K$ actually requires that the index vector for the form be in the same class as the index vector in the definition. Here the class of an index vector is determined by the index pair. The first 
construction will follow this idea in the case where $Q_{K}=L=(1)$ the $1 \times 1$ identity matrix. The problems with the class of the index vector will not arise until the general construction.

3.2. Symplectic theta function. We now introduce the function which will be used to create a symplectic modular form, namely the symplectic theta function

$$
\Theta_{\mathrm{Sp}}\left(Z,\left(\begin{array}{l}
U \\
V
\end{array}\right)\right)=\sum_{m \in \mathbb{Z}^{n}} e^{\pi i\left({ }^{t}(m+V) Z(m+V)-2^{t} m U-{ }^{t} V U\right)}
$$

where $Z$ is in $\mathfrak{h}^{(n)}$, and $U, V$ in $\mathbb{C}^{n}$ are fixed. $\Theta_{\mathrm{Sp}}(Z)$ is a symplectic modular form of weight $1 / 2$ with a multiplier system for $\Gamma_{\theta}^{(n)}$ where $\Gamma_{\theta}^{(n)}=\left\{M=\left(\begin{array}{cc}A & B \\ C & D\end{array}\right) \in \operatorname{Sp}_{2 n}(\mathbb{Z}) \mid A^{t} B, C^{t} D\right.$ have even diagonal entries $\}$. Specifically, this means

$$
\begin{aligned}
& \Theta_{\mathrm{Sp}}\left(\left(\begin{array}{cc}
A & B \\
C & D
\end{array}\right) \circ Z, M\left(\begin{array}{l}
U \\
V
\end{array}\right)\right) \\
& =\chi(M) \operatorname{det}(C Z+D)^{1 / 2} \Theta_{\mathrm{Sp}}\left(Z,\left(\begin{array}{l}
U \\
V
\end{array}\right)\right) \quad \forall M=\left(\begin{array}{cc}
A & B \\
C & D
\end{array}\right) \in \Gamma_{\theta}^{(n)}
\end{aligned}
$$

where $\chi(M)$ is an eighth root of unity depending only on the matrix $M$ and the value of the square root that is taken. This root of unity can be explicitly determined in certain cases using results of [10].

There is an alternate presentation of this theta function which changes the group under which this function transforms. Namely by replacing the $\pi i$ that appears in (12) by a $2 \pi i$ produces a function that does not transform properly under $\Gamma_{\theta}^{(n)}$ but under the group

$$
\Gamma_{0}^{(n)}(4)=\left\{\left(\begin{array}{cc}
A & B \\
C & D
\end{array}\right) \in \operatorname{Sp}_{2 n}(\mathbb{Z}) \mid C \text { has entries divisible by } 4\right\} .
$$

This type of group is more common in the literature, and makes calculations simpler to present; however it is quite a bit smaller than $\Gamma_{\theta}^{(n)}$ so the simplification comes at the price of a large number of transformation formulas. In this paper the " $2 \pi i$ " version will be used to simplify the statements of results and to simplify the construction however the construction with the " $i i$ " version works just as well.

3.3. Embeddings. The method of creating a symplectic theta function that transforms appropriately is to embed $\Gamma^{\mathrm{J}}\left(\mathcal{O}_{K}\right)$ into $\mathrm{Sp}_{8}(\mathbb{Z})$ and the space $\mathfrak{h}^{(\mathcal{Q})} \times \mathcal{Q}$ into $\mathfrak{h}^{(4)}$. We will introduce notation to make this easier to state. We set for $(\tau, z)$ in $\mathfrak{h}^{(\mathcal{Q})} \times \mathcal{Q}, \tau=x+y \kappa, z=u+v \kappa$. It should be 
noted that all of the following quaternionic embeddings are based on the same representation, i.e.

$$
a+b \kappa \in \mathcal{Q} \hookrightarrow\left(\begin{array}{cc}
a & i b \\
i \bar{b} & \bar{a}
\end{array}\right) \in M_{2}(\mathbb{C}) .
$$

Now embed the space $\mathcal{H}$ into $\mathfrak{h}^{(4)}$ by sending $(\tau, z)$ to

$$
Z=\left(\begin{array}{cccc}
x & i y & u & i v \\
i y & \bar{x} & i \bar{v} & \bar{u} \\
u & i \bar{v} & x^{\prime} & i y^{\prime} \\
i v & \bar{u} & i y^{\prime} & \bar{x}^{\prime}
\end{array}\right)
$$

There is an extra quaternionic upper half plane variable denoted $\tau^{\prime}=x^{\prime}+$ $y^{\prime} \kappa$, which was embedded in the lower right $2 \times 2$ corner of the matrix. The Fourier expansion will be formed with respect to the extra variable $x^{\prime}$, after creating the symplectic modular form. Note this matrix is clearly symmetric and not hermitian.

Another embedding allows a sum over rational integers instead of integers in $\mathcal{O}_{K}$, assume $\mathcal{O}_{K}=\left[\omega_{1}, \omega_{2}\right]_{\mathbb{Z}}$, and set

$$
W=\left(\begin{array}{ll}
\omega_{1} & \omega_{2} \\
\bar{\omega}_{1} & \bar{\omega}_{2}
\end{array}\right)
$$

This matrix $W$ converts rational integers into elements of $\mathcal{O}_{K}$, i.e. given $\lambda$ in $\mathcal{O}_{K}, \lambda=a_{1} \omega_{1}+a_{2} \omega_{2}$ with $a_{j}$ in $\mathbb{Z}$, then

$$
\left(\frac{\lambda}{\lambda}\right)=\left(\begin{array}{ll}
\omega_{1} & \omega_{2} \\
\bar{\omega}_{1} & \bar{\omega}_{2}
\end{array}\right)\left(\begin{array}{l}
a_{1} \\
a_{2}
\end{array}\right)
$$

Define $\widehat{W}=\left(\begin{array}{cc}W & 0 \\ 0 & W\end{array}\right)$ and create the symplectic theta function

$\Theta\left(Z,\left(\begin{array}{l}0 \\ 0\end{array}\right)\right)=\sum_{m \in \mathbb{Z}^{4}} e^{2 \pi i^{t} m}{ }^{t} \widehat{W} Z \widehat{W} m=\sum_{m \in \mathbb{Z}^{4}} e^{2 \pi i{ }^{t} m \tilde{Z} m} \quad$ where $\widetilde{Z}={ }^{t} \widehat{W} Z \widehat{W}$.

In order for this to be a symplectic theta function it is necessary to know that $\widetilde{Z}$ is in the space $\mathfrak{h}^{(4)}$. For this we need the following lemma.

LEMMA 1. Assume $V$ and $T$ are invertible complex matrices, then if $Z={ }^{t} \bar{T} V T={ }^{t} Z$ the imaginary part of $Z$ is positive definite if $\frac{1}{2 i}\left(V-{ }^{t} \bar{V}\right)$ is positive definite.

Proof. The imaginary part of $t \bar{T} V T$ is

$$
\frac{1}{2 i}\left[{ }^{t} \bar{T} V T-{ }^{t} T \overline{V T}\right]=\frac{1}{2 i}\left[{ }^{t} \bar{T} V T-{ }^{t} \bar{T}{ }^{t} \bar{V} T\right]
$$

since $Z$ is symmetric. Simplifying we find this is ${ }^{t} \bar{T}\left[\frac{1}{2 i}\left(V-{ }^{t} \bar{V}\right)\right] T$ and the expression in the parentheses is positive definite by assumption. Multiplying 
by ${ }^{t} \bar{T}$ and $T$ does not affect the positive definite nature of the expression in parentheses.

The converse of the lemma is also true but will be unnecessary in the construction.

To see that $\widetilde{Z}$ is in $\mathfrak{h}^{(4)}$, define

$$
S=\left(\begin{array}{llll}
0 & 1 & & \\
1 & 0 & & \\
& & 0 & 1 \\
& & 1 & 0
\end{array}\right) .
$$

Then $S Z S$ is the same matrix as $Z$ with the complex conjugate pairs switched in all of the variables. Similarly $S \widehat{W}$ is the same as the matrix $\widehat{W}$ with the complex conjugate pairs of rows switched which will be written as $\bar{W}$. Note $S^{2}=I_{4}$ and write ${ }^{t} \widehat{W} Z \widehat{W}={ }^{t} \widehat{W} S S Z \widehat{W}={ }^{t} \bar{W} S Z \widehat{W}$ and the imaginary part of $S Z$ is (using the notation $\tau=\left(x_{1}+i x_{2}\right)+y \kappa, z=$ $\left(u_{1}+i u_{2}\right)+\left(v_{1}+i v_{2}\right) \kappa$ and similarly for $\left.\tau^{\prime}\right)$,

$$
\left(\begin{array}{cccc}
y & -x_{2} & v_{1} & -u_{2} \\
x_{2} & y & u_{2} & v_{1} \\
v_{1} & -u_{2} & y^{\prime} & -x_{2}^{\prime} \\
u_{2} & v_{1} & x_{2}^{\prime} & y^{\prime}
\end{array}\right) .
$$

This matrix can be forced to be positive definite simply by picking the $y^{\prime}$ variables to be large enough based on the other variables in the matrix. So some conditions on the extra upper half plane variables forces the imaginary part of the matrix in (15) to be positive definite and therefore the matrix ${ }^{t} \widehat{W} Z \widehat{W}={ }^{t} \bar{W} S Z \widehat{W}$ has positive definite imaginary part and since it is obviously symmetric it is in $\mathfrak{h}^{(4)}$.

Expanded out, this theta function looks like $\Theta(Z)=\Theta\left(\tau, z, \tau^{\prime}\right)$

$$
\Theta\left(\tau, z, \tau^{\prime}\right)=\sum_{\lambda_{1}, \lambda_{2} \in \mathcal{O}_{K}} e\left[\lambda_{1} \tau \lambda_{1}+2 \lambda_{1} z \lambda_{2}\right] e\left[\lambda_{2} \tau^{\prime} \lambda_{2}\right]
$$

This first part mimics the classical Jacobi theta functions where one thinks of the $\lambda_{2}$ as fixed and the quadratic form $Q=(1)$. In fact the first line is the Fourier expansion with respect to the $\tau^{\prime}$, so the Fourier expansion with respect to $\tau^{\prime}$ may be written

$$
\Theta\left(\tau, z, \tau^{\prime}\right)=\sum_{\lambda_{2} \in \mathcal{O}_{K}} \Phi_{1 / 2, \lambda_{2}}(\tau, z) e\left[\lambda_{2} \tau^{\prime} \lambda_{2}\right]
$$

where

$$
\Phi_{1 / 2, \lambda_{2}}(\tau, z)=\sum_{\lambda_{1} \in \mathcal{O}_{K}} e\left[\lambda_{1} \tau \lambda_{1}+2 \lambda_{1} z \lambda_{2}\right]
$$


For a proper Fourier expansion the coefficients corresponding to $\lambda_{2}$ and $-\lambda_{2}$ should be grouped together since they are both part of the same Fourier coefficient. However, since they are identical we leave them separate to mimic Jacobi's original examples.

The next step in the construction is to embed the Jacobi group $\Gamma^{\mathrm{J}}\left(\mathcal{O}_{K}\right)$ into $\mathrm{Sp}_{8}(\mathbb{Z})$ and show that the action on the embedded variables $(\tau, z)$ as an element of $\mathfrak{h}^{(4)}$ is the claimed group action on the space. First define the notation $\breve{d}(\alpha)$, for $\alpha$ in $K$, by $\check{d}(\alpha)=\left(\begin{array}{cc}\alpha & 0 \\ 0 & \alpha\end{array}\right)$.

The embeddings of the elements of the Jacobi group are:

$$
\begin{aligned}
& \left(\begin{array}{ll}
\alpha & \beta \\
\gamma & \delta
\end{array}\right) \in \operatorname{Sl}_{2}\left(\mathcal{O}_{K}\right) \hookrightarrow\left(\begin{array}{cccc}
\check{d}(\alpha) & 0 & \check{d}(\beta) & 0 \\
0 & I_{2} & 0 & 0 \\
\check{d}(\gamma) & 0 & \check{d}(\delta) & 0 \\
0 & 0 & 0 & I_{2}
\end{array}\right)=\left(\begin{array}{cc}
A & B \\
C & D
\end{array}\right), \\
& {[\lambda, \mu] \in \mathcal{O}_{K}^{2} \hookrightarrow\left(\begin{array}{cccc}
I_{2} & 0 & 0 & \check{d}(\mu) \\
\check{d}(\lambda) & I_{2} & \check{d}(\mu) & \check{d}(\lambda \mu) \\
0 & 0 & I_{2} & \check{d}(-\lambda) \\
0 & 0 & 0 & I_{2}
\end{array}\right)=\left(\begin{array}{cc}
A^{\prime} & B^{\prime} \\
C^{\prime} & D^{\prime}
\end{array}\right) .}
\end{aligned}
$$

With these embeddings

$$
\begin{aligned}
& \left(\begin{array}{ll}
\alpha & \beta \\
\gamma & \delta
\end{array}\right) \circ\left(\tau, z, \tau^{\prime}\right) \\
& \hookrightarrow\left(\begin{array}{cccc}
\alpha x+\beta & i \alpha y & \alpha u & i \alpha v \\
\bar{\alpha} y & \overline{\alpha x}+\bar{\beta} & i \overline{\alpha v} & \overline{\alpha u} \\
u & i \bar{v} & x^{\prime} & i y^{\prime} \\
i v & \bar{u} & i y^{\prime} & \bar{x}^{\prime}
\end{array}\right) \circ\left(\begin{array}{cccc}
\gamma x+\delta & \gamma y & \gamma u & i \gamma v \\
i \bar{\gamma} y & \overline{\gamma x}+\bar{\beta} & i \overline{\gamma v} & \overline{\gamma u} \\
0 & 0 & I & 0 \\
0 & 0 & 0 & I
\end{array}\right)^{-1} .
\end{aligned}
$$

It may be verified that this action with these embeddings takes the variables

$$
\begin{gathered}
\tau \rightarrow(\alpha \tau+\beta)(\gamma \tau+\delta)^{-1}, \quad z \rightarrow(\tau \gamma+\delta)^{-1} z \\
\tau^{\prime} \rightarrow \tau^{\prime}-(u+\bar{v} \kappa)(\gamma \tau+\delta)^{-1} \gamma(u+v \kappa)
\end{gathered}
$$

where the quaternionic factors are still embedded using (14). These are exactly the prescribed actions of the matrix $\left(\begin{array}{ll}\alpha & \beta \\ \gamma & \delta\end{array}\right)$ on the space $\mathcal{H}$, and the extra variable $\tau^{\prime}$ is transformed by subtracting the exponential transformation factor from formula (10).

It should be noted that the action $\left(\begin{array}{ll}A & B \\ C & D\end{array}\right) \circ Z$ is the same as the action

$$
\left(\begin{array}{cc}
{ }^{t} W A^{t} W^{-1} & { }^{t} W B W \\
W^{-1} C^{t} W^{-1} & W^{-1} D W
\end{array}\right) \circ \widetilde{Z}
$$

(recall $\left.\widetilde{Z}={ }^{t} W Z W\right)$. In order for this embedding of $\Gamma^{\mathrm{J}}\left(\mathcal{O}_{K}\right)$ to act on the 
symplectic theta function it is necessary that these embedded matrices be in $\mathrm{Sp}_{8}(\mathbb{Z})$ or to be more precise $\Gamma_{0}^{(4)}(4)$. The next step is to calculate the conditions on $\alpha, \beta, \gamma, \delta$ that will force

$$
\left(\begin{array}{cc}
\check{A} & \check{B} \\
\check{C} & \check{D}
\end{array}\right)=\left(\begin{array}{cc}
{ }^{t} W A^{t} W^{-1} & { }^{t} W B W \\
W^{-1} C^{t} W^{-1} & W^{-1} D W
\end{array}\right) \in \Gamma_{0}^{(4)}(4) .
$$

Since $W$ is a matrix of integers in the field and contains a basis for the ring of integers $\mathcal{O}_{K}$ the inverse matrix $W^{-1}$ is a basis for $\delta_{K}^{-1}$, the inverse different of the field $K$. It is easy to check that each of the entries in the matrices $\check{A}, \check{B}, \check{C}, \check{D}$ is a trace of an element of $K$. Since $W^{-1}$ is a basis for the inverse different, as long as $\alpha, \beta, \delta$ are in $\mathcal{O}_{K}$ the entries of $\check{A}, \check{B}, \check{D}$ will be traces of elements of $\delta_{K}^{-1}$ and therefore rational integers. In order for $\check{C}$ to be integral it is required that $\gamma$ be in $\delta_{K}$, i.e. $\gamma$ must be in the different. This is because the entries of $\check{C}$ are traces of elements in $\delta_{K}^{-2}(\gamma)$ and by the definition of the inverse different, an element of the inverse different has integral trace. So as long as $\gamma$ is in $\delta_{K}, \check{C}$ will have entries that are traces of elements in $\delta_{K}^{-1}$. In order for this matrix to be in $\Gamma_{0}^{(4)}(4)$ it is sufficient to require that 4 divide $\gamma$. Therefore as long as $\left(\begin{array}{ll}\alpha & \beta \\ \gamma & \delta\end{array}\right) \in \Gamma_{0}\left(4 \delta_{K}\right)$ the matrix $\left(\begin{array}{ll}\check{A} & \check{B} \\ \check{C} & \check{D}\end{array}\right) \in \Gamma_{0}^{(4)}(4)$.

It is easy to verify that

$$
\operatorname{det}(\check{C} \widetilde{Z}+\check{D})=\operatorname{det}(C Z+D)=\mathcal{N}(\gamma \tau+\delta)
$$

Therefore matching the Fourier coefficients of the symplectic theta function and the transformed version by (13) and using the fact that $\Theta(Z)$ is a symplectic modular form, the coefficients satisfy

$$
\begin{aligned}
& \Phi_{1 / 2, \lambda_{2}}\left(\left(\begin{array}{cc}
\alpha & \beta \\
\gamma & \delta
\end{array}\right) \circ(\tau, z)\right) \\
& =\chi(M) \mathcal{N}(\gamma \tau+\delta)^{1 / 2} e\left[\lambda_{2}(u+\bar{v} \kappa)(\gamma \tau+\delta)^{-1} \gamma(u+v \kappa) \lambda_{2}\right] \Phi_{1 / 2, \lambda_{2}}(\tau, z) .
\end{aligned}
$$

This agrees with the formula (10) given in the definition of a Jacobi form over $K$ of weight $1 / 2$, index $\lambda_{2}^{2}$ and index vector $\left(\lambda_{2}\right)$.

The second transformation law is proved by looking at the actions of $[\lambda, \mu]$ in $\mathcal{O}_{K}^{2}$ embedded in $\operatorname{Sp}_{8}(\mathbb{Z})$ and its action on the variables $\left(\tau, z, \tau^{\prime}\right)$ and comparing Fourier coefficients. The embedded $[\lambda, \mu]$ matrix takes

$$
\tau \rightarrow \tau, \quad z \rightarrow z+\tau \lambda+\mu, \quad \tau^{\prime} \rightarrow \tau^{\prime}+\lambda \tau \lambda+2 \lambda z+\lambda \mu .
$$

It is trivial to see that $\operatorname{det}\left(C^{\prime} \widetilde{Z}+D^{\prime}\right)=1$, and therefore by the same argument as above that by matching the Fourier coefficients on both sides of the symplectic theta function transformation formula (13) one extracts the transformation formulas for these Fourier coefficients. These formulas are exactly those given in (11) of the definition for a Jacobi form of weight 
$1 / 2$, index $\lambda_{2}^{2}$ and index vector $\left(\lambda_{2}\right)$. It is also easy to check that in order for

$$
\left(\begin{array}{cc}
{ }^{t} W A^{\prime} W^{-1} & { }^{t} W B^{\prime} W \\
W^{-1} C^{\prime} W^{-1} & W^{-1} D^{\prime} W
\end{array}\right) \in \Gamma_{0}^{(4)}(4)
$$

the only requirement is that $[\lambda, \mu]$ be in $\mathcal{O}_{K}^{2}$ since $W^{-1} C^{\prime} W^{-1}=0$. Therefore by this construction, one is able to produce Jacobi forms on $\Gamma_{0}\left(4 \delta_{K}\right) \ltimes$ $\mathcal{O}_{K}^{2}$ of weight $1 / 2$, index $\lambda_{2}^{2}$ and index vector $\left(\lambda_{2}\right)$ for all $\lambda_{2}$ in $\mathcal{O}_{K}$.

4. General construction with quadratic forms. The initial example given above created Jacobi forms of weight $1 / 2$, index $\lambda^{2}$, and index vector $(\lambda)$ for all $\lambda$ in $\mathcal{O}_{K}$. We now present a more general construction which will create forms of arbitrary half integral weight, more general indexes in $\mathcal{O}_{K}$ and much more general index vectors. The forms which will be created here are going to be generalizations of the Jacobi theta functions for a positive definite quadratic form over the rational integers of the type that appear in [4].

For this general construction we are using the " $2 \pi i$ " version of the symplectic theta function which will force all of the transformation formulas onto a $\Gamma_{0}$ congruence subgroup instead of the $\Gamma_{\theta}$ type congruence subgroups. The use of the " $\pi i$ " version is almost identical with the only difference being the subgroup of the symplectic group under which the functions transform.

4.1. Quadratic forms. There are some facts about quadratic forms which will be useful in order to create Jacobi forms. A quadratic form is a symmetric function

$$
Q: \mathbb{C}^{n} \times \mathbb{C}^{n} \rightarrow \mathbb{C}
$$

which will be represented as a matrix so

$$
Q(\vec{a}, \vec{b})={ }^{t} \vec{a} Q \vec{b}, \quad Q \in M_{n}(\mathbb{C}), \vec{a}, \vec{b} \in \mathbb{C}^{n} .
$$

A symmetric quadratic form is such that

$$
Q(\vec{a}, \vec{b})=Q(\vec{b}, \vec{a})
$$

so it is necessary that the matrix $Q$ be symmetric, ${ }^{t} Q=Q$. Note that it is meaningless to talk about a quadratic form over the complex numbers being positive definite, however in the case of complex entries, a quadratic form will be required to have non-zero determinant. Given such a matrix there will always be an upper triangular matrix $L \in M_{n}(\mathbb{C})$ such that $Q={ }^{t} L L$, $L=\left(l_{i, j}\right)_{1 \leq i, j \leq n}$ with $l_{i, j}=0$ for $j<i$.

For this construction, the quadratic form $Q$ is in $M_{l}\left(\mathcal{O}_{K}\right)$, assume that $Q=\left(q_{s, t}\right)_{1 \leq s, t \leq l}$ so all of the entries are integers in the number field. Since $Q$ has entries in $K$, it is reasonable to define the conjugates $\bar{Q}=\left(\bar{q}_{s, t}\right)_{1 \leq s, t \leq l}$ and define $\bar{L}$ by $\bar{Q}={ }^{t} \bar{L} \bar{L}$. 
4.2. The general theta functions. Given a quadratic form over $\mathcal{O}_{K}$ as in the previous section, we will create a theta function of the form, for $b$ in $\mathcal{O}_{K}^{l}$,

$$
\theta_{Q, b}(\tau, z)=\sum_{a \in \mathcal{O}_{K}^{l}} e\left[{ }^{t} a^{t} L \tau L a+2{ }^{t} a^{t} L z L b\right] .
$$

These functions will be produced as before by creating a symplectic theta function and choosing the appropriate Fourier coefficients. This construction and the verification of the construction will prove the following theorem.

TheOREM. Let $K$ be an imaginary quadratic number field as usual. Let $Q$ be an $l \times l$ quadratic form with entries in $\mathcal{O}_{K}$, and let $\mathfrak{g}$ be the greatest common divisor of the minors of $Q$, and let $Q={ }^{t} L L$. Then the function

$$
\Theta_{l / 2, \vec{m}, \mathbf{m}}(\tau, z)=\sum_{\vec{m}, \mathbf{m}} \theta_{Q, b}(\tau, z)
$$

is a Jacobi form of weight $l / 2$, index $Q(b)$, and index vector $L b$ for the subgroup $\Gamma_{0}\left(4 \delta_{K} \mathfrak{g}^{-2} \operatorname{det}(Q)^{2}\right) \ltimes \mathcal{O}_{K}^{2} \subseteq \Gamma^{\mathrm{J}}\left(O_{K}\right)$. The sum over $\vec{m}, \mathbf{m}$ means to sum over all vectors $b \in \mathcal{O}_{K}^{l}$ such that $Q(b)=\mathbf{m}$ and ${ }^{t} \overline{b^{t} L} L b={ }^{t} \vec{m} \vec{m}$.

In the case where there is only one $b$ in $\mathcal{O}_{K}^{l}$ in the sum, this is the natural generalization of the Jacobi theta function. However, for a general index pair $\{\mathbf{m}, \vec{m}\}$ all that can be shown is that the sum is over a finite number of $b$. Note $\delta_{K}$ is the different of $K$ as in the earlier notation.

Begin by introducing notation. For $a \in \mathbb{C}$ or any complex variable let

$$
d_{l}(a)=\left(\begin{array}{cccc}
a & 0 & \cdots & \\
0 & a & & \\
\vdots & & \ddots & \\
& & & a
\end{array}\right)
$$

which is an $l \times l$ matrix. Now define the $l \times 2 l$ matrix

$$
W=\left(\begin{array}{cccccccc}
\omega_{1} & \omega_{2} & 0 & 0 & \ldots & & & \\
0 & 0 & \omega_{1} & \omega_{2} & 0 & \ldots & & \\
& & & & & \ddots & 0 & 0 \\
& & & & & 0 & \omega_{1} & \omega_{2}
\end{array}\right)
$$

and define the matrices $\widehat{W}$ and $\widehat{L}$ to be

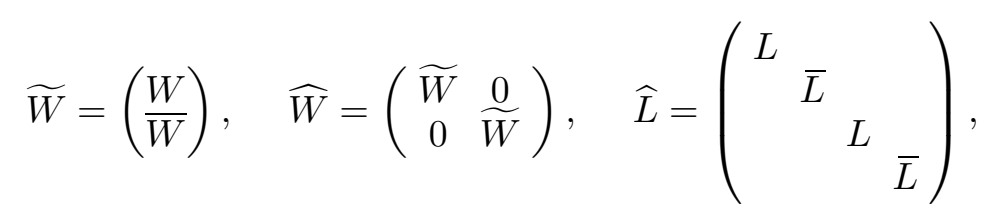

where the $\bar{L}$ is the matrix such that $\bar{Q}={ }^{t} \bar{L} \bar{L}$. The variable for the symplectic 
theta function is defined as

$$
\widetilde{Z}=\left(\begin{array}{cccc}
d_{l}(x) & i d_{l}(y) & d_{l}(u) & i d_{l}(v) \\
i d_{l}(y) & d_{l}(\bar{x}) & i d_{l}(\bar{v}) & d_{l}(\bar{u}) \\
d_{l}(u) & i d_{l}(\bar{v}) & d_{l}\left(x^{\prime}\right) & i d_{l}\left(y^{\prime}\right) \\
i d_{l}(v) & d_{l}(\bar{u}) & i d_{l}\left(y^{\prime}\right) & d_{l}\left(\bar{x}^{\prime}\right)
\end{array}\right)
$$

Now create the symplectic theta function

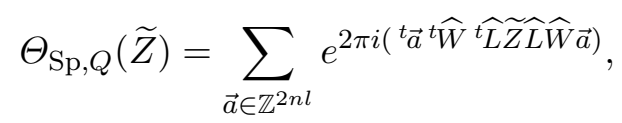

but for this to be a symplectic theta function it must be verified that ${ }^{t} \widehat{W}{ }^{t} \widehat{L} \widetilde{Z} \widehat{L} \widehat{W}$ is in $\mathfrak{h}^{(4 l)}$ or at least that one can pick the extra upper half plane variables in order to put this matrix into the symplectic upper half space. The matrix is obviously symmetric by construction therefore it remains to show it has positive definite imaginary part. By the same reasoning as earlier consider the matrix $\widetilde{S}$

$$
\left(\begin{array}{cccc}
0 & I_{l} & & \\
I_{l} & 0 & & \\
& & 0 & I_{l} \\
& & I_{l} & 0
\end{array}\right)
$$

which has the same effect as before, it switches the complex conjugate rows of the matrix $\widehat{L} \widehat{W}$ which is denoted $\overline{L W}=\widetilde{S} \widehat{L} W$. Therefore in order to show this matrix

$$
{ }^{t} \widehat{W}^{t} \widehat{L} \widetilde{Z} \widehat{L} \widehat{W}
$$

has positive definite imaginary part, note $\widetilde{S}^{2}=I_{4 l}$, and consider

$$
{ }^{t} \widehat{W}^{t} \widehat{L} \widetilde{Z} \widehat{L} \widehat{W}={ }^{t} \widehat{W}^{t} \widehat{L} S S \widetilde{Z} \widehat{L} \widehat{W}={ }^{t} W^{t} L \widetilde{S} \widetilde{Z} \widehat{L} W
$$

and if $\widetilde{S} \widetilde{Z}$ has positive definite imaginary part then so does (21) by the earlier lemma. The imaginary part of $\widetilde{S} \widetilde{Z}$ is

$$
\left(\begin{array}{cccc}
d_{l}(y) & d_{l}\left(-x_{2}\right) & d_{l}\left(v_{1}\right) & d_{l}\left(-u_{2}\right) \\
d_{l}\left(x_{2}\right) & d_{l}(y) & d_{l}\left(u_{2}\right) & d_{l}\left(v_{1}\right) \\
d_{l}\left(v_{1}\right) & d_{l}\left(-u_{2}\right) & d_{l}\left(y^{\prime}\right) & d_{l}\left(-x_{2}^{\prime}\right) \\
d_{l}\left(u_{2}\right) & d_{l}\left(v_{1}\right) & d_{l}\left(x_{2}^{\prime}\right) & d_{l}\left(y^{\prime}\right)
\end{array}\right)
$$

using the earlier notation $\tau=\left(x_{1}+i x_{2}\right)+y \kappa, z=\left(u_{1}+i u_{2}\right)+\left(v_{1}+i v_{2}\right) \kappa$ and similarly for $\tau^{\prime}$. This matrix is just $l$ copies of the matrix in (15) and since we can pick the $y^{\prime}$ variable so that (15) is positive definite, the same choice of $y^{\prime}$ will force the above matrix to be positive definite.

Expanded out, this symplectic theta function (20) looks like

$$
\Theta_{\mathrm{Sp}, Q}(\widetilde{Z})=\Theta_{\mathrm{Sp}, Q}\left(\tau, z, \tau^{\prime}\right)
$$


and

$$
\begin{aligned}
\Theta_{\mathrm{Sp}, Q}\left(\tau, z, \tau^{\prime}\right) & =\sum_{a, b \in \mathcal{O}_{K}^{l}} e\left[\left({ }^{t} a{ }^{t} L \tau L a+2{ }^{t} a{ }^{t} L z L b\right)\right] e\left[\left({ }^{t}{ }^{t} L \tau_{j}^{\prime} L b\right)\right] \\
& =\sum_{b \in \mathcal{O}_{K}^{l}} \theta_{Q, b}(\tau, z) e\left[{ }^{t} b^{t} L \tau^{\prime} L b\right]
\end{aligned}
$$

where

$$
\theta_{Q, b}(\tau, z)=\sum_{a \in \mathcal{O}_{K}^{l}} e\left[\left({ }^{t} a{ }^{t} L \tau L a+2{ }^{t} a{ }^{t} L z L b\right)\right] .
$$

This last representation shows how the Jacobi theta functions $\theta_{Q, b}$ arise as the Fourier coefficients with respect to the $\tau^{\prime}$ variable. However, there may be a number of vectors $b$ in $\mathcal{O}_{K}^{l}$ which are all part of the same Fourier coefficient because they all produce the same index $Q(b)$. In particular this last representation may be written as

$$
\Theta_{\mathrm{Sp}, Q}\left(\tau, z, \tau^{\prime}\right)=\sum_{m \in \mathcal{O}_{K}} \sum_{\left(b \in \mathcal{O}_{K}^{l} \mid Q(b)=m\right)} \theta_{Q, b}(\tau, z) e\left[{ }^{t} b^{t} L \tau L b\right] .
$$

Some of these Jacobi theta functions will be separated from each other so that they will be shown to transform as Jacobi forms independent of the other Jacobi theta functions in the coefficient. Some of these $\theta_{Q, b}$ will still be grouped together in the Fourier coefficient but it will be shown that they as a group transform correctly according to the definition of Jacobi forms over $K$ with a given index vector.

Before we can fully state the transformation formulas of these Fourier coefficients it is necessary to examine the structure of the coefficient. The formula above (24) is a Fourier expansion with respect to $x^{\prime}$, but the $y^{\prime}$ are not part of the expansion (because the form is not analytic). They are grouped in simply as a matter of notational convenience. However, it is possible to assume that the non-complex parts of the quaternionic variables, the $y^{\prime}$, are part of the Fourier expansion at least in order to show the transformation formulas. It was already noted that all of the Jacobi theta functions with index vectors $b$ such that $Q(b)=\mathbf{m}$ are summed to make the $\mathbf{m}$ th coefficient. It is possible to separate these into classes based on the associated index pair $\left(Q(b),{ }^{t} b^{t} L L b\right)$. Since there are only a finite number of vectors $b$ which produce the same index there is a smallest ${ }^{t} \overline{b^{t} L} L b$ because these are all real numbers. The sum of these Jacobi theta functions with the same index pair can then be separated from the rest of the Fourier coefficient by letting the $y^{\prime}$ go to infinity. This will force the theta functions associated with the larger ${ }^{t} b^{t} L L b$ to go to zero faster. Now that this portion of the Fourier coefficient is separated it may be shown to be a Jacobi form over $K$ of weight $l / 2$, index $Q(b)$ and index vector $L b$ where $b$ is the vector associated with any 
one of the theta functions in the class. Similarly once this class of Jacobi theta functions is shown to be a Jacobi form one can take the next smallest ${ }^{t} \overline{b^{t} L} L b$ and repeat the process thus separating out all of the different classes of theta functions.

4.3. Transformation formulas. Now it is necessary to show this sum over a class of Jacobi theta functions transforms correctly. As before, it is necessary to extend the group embeddings from the first example so that the embedded group will act on $\widetilde{Z}$. We generalize the earlier definition of $\breve{d}(\alpha)$ to $\breve{d}_{l}(\alpha)$, for $\alpha$ in $\mathcal{O}_{K}$,

$$
\check{d}_{l}(\alpha)=\left(\begin{array}{cc}
d_{l}(\alpha) & 0 \\
0 & d_{l}(\bar{\alpha})
\end{array}\right) .
$$

Then the embeddings of the elements of $\Gamma^{\mathrm{J}}\left(\mathcal{O}_{K}\right)$ are:

$$
\begin{gathered}
\left(\begin{array}{cc}
\alpha & \beta \\
\gamma & \delta
\end{array}\right) \in \operatorname{Sl}_{2}\left(\mathcal{O}_{K}\right) \hookrightarrow\left(\begin{array}{cccc}
\check{d}_{l}(\alpha) & 0 & \check{d}_{l}(\beta) & 0 \\
0 & I_{2 l} & 0 & 0 \\
\check{d}_{l}(\gamma) & 0 & \check{d}_{l}(\delta) & 0 \\
0 & 0 & 0 & I_{2 l}
\end{array}\right)=\left(\begin{array}{cc}
A & B \\
C & D
\end{array}\right), \\
{[\lambda, \mu] \in \mathcal{O}_{K}^{2} \hookrightarrow\left(\begin{array}{cccc}
I_{2 l} & 0 & 0 & \check{d}_{l}(\mu) \\
\check{d}_{l}(\lambda) & I_{2 l} & \check{d}_{l}(\mu) & \check{d}_{l}(\lambda \mu) \\
0 & 0 & I_{2 l} & \check{d}_{l}(-\lambda) \\
0 & 0 & 0 & I_{2 l}
\end{array}\right)=\left(\begin{array}{cc}
A^{\prime} & B^{\prime} \\
C^{\prime} & D^{\prime}
\end{array}\right) .}
\end{gathered}
$$

Set $T=\widehat{L} \widehat{W}$. It should be noted that the action of $\left(\begin{array}{ll}\alpha & \beta \\ \gamma & \delta\end{array}\right) \circ(\vec{\tau}, \vec{z})$ is the same as the action of

$$
\left(\begin{array}{cc}
{ }^{t} T A^{t} T^{-1} & { }^{t} T B T \\
T^{-1} C^{t} T^{-1} & T^{-1} D T
\end{array}\right) \circ{ }^{t} T \widetilde{Z} T
$$

and similarly for the embedded $[\lambda, \mu]$ matrix. In fact the actions are exactly the same as those in the first construction in (17) and (18), since these embeddings are simply $l$ copies of the embeddings in the first construction. So, by introducing a quadratic form the actions have not changed but it is still necessary to check that this matrix

$$
\left(\begin{array}{cc}
\widetilde{A} & \widetilde{B} \\
\widetilde{C} & \widetilde{D}
\end{array}\right)=\left(\begin{array}{cc}
{ }^{t} T A^{t} T^{-1} & { }^{t} T B T \\
T^{-1} C^{t} T^{-1} & T^{-1} D T
\end{array}\right)
$$

is in $\operatorname{Sp}_{8 l}(\mathbb{Z})$ or particularly $\Gamma_{0}^{(8 l)}(4)$, whenever $\left(\begin{array}{cc}\alpha & \beta \\ \gamma & \delta\end{array}\right)$ is in a subgroup of $\mathrm{Sl}_{2}\left(\mathcal{O}_{K}\right)$ and similarly for the embedded $[\lambda, \mu]$ matrix.

In order to check that this embedded group matrix is actually in $\operatorname{Sp}_{8 l}(\mathbb{Z})$ consider the entries of the matrices $\widetilde{A}, \widetilde{B}, \widetilde{C}, \widetilde{D}$ which with a little computa- 
tion are all traces of elements in $K$. One fact which is helpful here is that the entries of $Q^{-1}$ are given in terms of determinants of minors divided by the determinant of $Q$. Therefore if $\mathfrak{g}$ is the ideal generated by the principal minors of $Q$ then all of the entries of $Q^{-1}$ are divisible by $\mathfrak{g d e t}(Q)^{-1}$. The entries in $\widetilde{A}, \widetilde{D}$ are all traces of elements from the ideal generated by $\delta_{K}^{-1}$ the traces of these elements are by definition all in $\mathbb{Z}$. The entries of $\widetilde{B}$ are all traces of elements in an ideal contained in the integers $O_{K}$ so they are rational integers. The entries of the matrix $\widetilde{C}$ are all traces of elements from the ideal $\delta_{K}^{-2} \mathfrak{g}^{2}(\operatorname{det}(Q))^{-2}$ and therefore in order for all of these entries to be rational integers we require that $\gamma$ be in $\delta_{K} \operatorname{det}(Q)^{2} \mathfrak{g}^{-2}$. Now in order for this matrix to be in $\Gamma_{0}^{(8 l)}(4)$ it is also necessary that all of the entries of $\widetilde{C}$ be divisible by 4 , therefore if $\gamma$ is in $4 \delta_{K} \operatorname{det}(Q)^{2} \mathfrak{g}^{-2}$ then the embedded matrix is in $\Gamma_{0}^{(8 l)}(4)$.

So far, the construction has produced a symplectic theta function which has combinations of Jacobi theta functions of a quadratic form $Q$ over the field $K$ as its Fourier coefficients and these may be separated into classes based on the index pair. In the last section it was shown that the embedded $\Gamma^{\mathrm{J}}\left(\mathcal{O}_{K}\right)$ acted correctly on the embedded version of the space $\mathcal{H}$, and it is easy to check that the actions on the $\tau, z, \tau^{\prime}$ are the same as above. It is also easy to check that $\operatorname{det}(C \widetilde{Z}+D)=\mathcal{N}(c \tau+d)^{l}$ as previously defined. Therefore since $\Theta_{\mathrm{Sp}, Q}$ is a symplectic modular form, by using (13) we see that for all $M=\left(\begin{array}{ll}A & B \\ C & D\end{array}\right) \in \Gamma_{0}^{(8 l)}(4)$,

$$
\Theta_{\mathrm{Sp}, Q}(M \circ \widetilde{Z})=\chi(M) \operatorname{det}(C \widetilde{Z}+D)^{1 / 2} \Theta_{\mathrm{Sp}, Q}(\widetilde{Z}) .
$$

Expanding this, we have

$$
\sum_{a \in \mathbb{Z}^{4 l}} e\left[2 \pi i^{t} a^{t} T(M \circ \widetilde{Z}) T a\right]=\chi(M) \mathcal{N}(c \tau+d)^{l / 2} \sum_{a \in \mathbb{Z}^{4 l}} \exp \left(2 \pi i^{t} a^{t} T \widetilde{Z} T a\right) .
$$

We expand out the right hand side of this equation as in the previous calculations, and look at the Fourier expansion (24) as in the earlier section. Since each

$$
\tau^{\prime} \rightarrow \tau^{\prime}-(u+\bar{v} \kappa)(\gamma \tau+\delta)^{-1} \gamma(u+v \kappa),
$$

we get the exponential part of the transformation formulas by matching the Fourier coefficients in (24), and by replacing $\tau^{\prime}$ as in (26) where the index for the Jacobi theta function is given by ${ }^{t} b^{t} L L b=Q(b)$ and the index vector is now given by $(L b)$.

Similarly, the embedded $[\lambda, \mu] \in \mathcal{O}_{K}^{2}$ matrix takes

$$
\begin{aligned}
\tau & \rightarrow \tau, \quad z \rightarrow z+\tau \lambda+\mu, \\
\tau^{\prime} & \rightarrow \tau^{\prime}+\lambda \tau \lambda+2 \lambda z+\lambda \mu .
\end{aligned}
$$


Also the action of

$$
\left(\begin{array}{cc}
\widehat{A} & \widehat{B} \\
\widehat{C} & \widehat{D}
\end{array}\right)=\left(\begin{array}{cc}
{ }^{t} T A^{\prime} t T^{-1} & { }^{t} T B^{\prime} T \\
T^{-1} C^{\prime} T^{-1} & T^{-1} D^{\prime} T
\end{array}\right)
$$

on ${ }^{t} T \widetilde{Z} T$ is the same as the action of $[\lambda, \mu]$ on $(\tau, z)$. It is trivial to see that $\operatorname{det}(\widehat{C} \widetilde{Z}+\widehat{D})=1$ and it is known that $\chi(M)=1$ in this case, and therefore we get the transformation formulas by the same argument as above, i.e. by matching the Fourier coefficients and pulling out the transformation formulas for these Fourier coefficients. So this calculation has verified that the $m$ th Fourier coefficient is in fact Jacobi forms of weight $l / 2$, index $Q(b)=$ $m$, and index vector $L b$.

\section{References}

[1] M. Eichler and D. Zagier, The Theory of Jacobi Forms, Birkhäuser, Boston, 1985.

[2] V. Gritsenko, Jacobi functions and Euler products for Hermitian modular forms, Zap. Nauchn. Sem. Leningrad. Otdel. Mat. Inst. Steklov. (LOMI) 183 (1990), $77-123$.

[3] K. Haverkamp, Hermitian Jacobi forms, Results Math. 29 (1996), 78-89.

[4] C. G. J. Jacobi, Fundamenta nova theoriae functionum ellipticarum, Königsberg, 1829.

[5] A. Krieg, The Maass space on the Hermitian half-space of degree 2, Math. Ann. 289 (1991), 663-681.

[6] -, Jacobi forms of several variables and the Maass space, J. Number Theory 56 (1996), 242-255.

[7] O. Richter, Theta functions of quadratic forms, PhD thesis, University of California, San Diego, La Jolla, CA 92093, June 1999.

[8] H. Skogman, Jacobi forms over number fields, PhD thesis, University of California, San Diego, La Jolla, CA 92093, June 1999.

[9] - Jacobi forms over totally real fields, to appear.

[10] H. Stark, On the transformation formula for the symplectic theta function and applications, J. Fac. Sci. Univ. Tokyo Sect. 1A 29 (1982), 1-12.

Department of Mathematics

University of Southern California

DRB 155

1042 W. 36th Place

Los Angeles, CA 90089-1113, U.S.A.

E-mail: skogman@math.usc.edu 